\title{
Les modes de régulation du système hospitalier L'exemple de la mise en œuvre des SROS III
}

\section{Hervé Michel a* et Alain Jourdain ${ }^{b}$}

a Docteur en science politique, MADoPA, 2, rue Gustave Eiffel, 10430 Rosières près Troyes

${ }^{b}$ Professeur de politiques de santé et planification, École des Hautes Etudes en Santé Publique, Av. du Pr Léon Bernard, 35043 Rennes Cedex

\section{Résumé}

La confrontation des logiques techniques et politiques est au centre de la planification hospitalière. Cela apparaît aussi bien au niveau des territoires de santé infra-régionaux qu'au niveau plus général des modes d'élaboration des schémas régionaux de l'organisation sanitaire (SROS) par les agences régionales de l'hospitalisation (ARH).

Plus précisément, l'analyse de la mise en œuvre des SROS III met clairement en évidence le rôle de l'ARH en tant qu'instance d'articulation, dans le calme ou dans la crise, des logiques techniques, financières et politiques portées par les différents acteurs régionaux. Elle permet aussi de cerner les contours d'un mode de régulation à dominante horizontale et régionale, renforcé par la « menace » d'une régulation verticale et nationale, c'est-à-dire d'arbitrages ministériels sous la pression des acteurs locaux. Les résultats de ces observations sont discutés au regard des modèles de régulation croisée et institutionnalisée.

Cet article apporte ainsi un éclairage sur le fonctionnement de la partie déconcentrée d'un État régulateur, qui « gouverne à distance » en définissant les cadres de l'organisation sanitaire et en laissant aux ARH une autonomie pour les mettre en œuvre en fonction des contextes régionaux.

(c) 2011 IDMP/Lavoisier SAS. Tous droits réservés

Mots clés : hôpital, planification, régionalisation, $\mathrm{SROS}, \mathrm{ARH}, \mathrm{ARS}$, politiques de santé, territoire de santé, État régulateur, régulation, système hospitalier.

\section{Abstract}

Modes of Regulation of the French Hospital System: the example of the SROS III. The confrontation of technical and political rationales is at the centre of hospital planning. This appears both at the level of infra-regional health territories and at the more general

*Auteur correspondant : herve.michel@madopa.fr doi:10.3166/pmp.28.279-309 @ 2011 IDMP/Lavoisier SAS. Tous droits réservés 
level of regional health organisation planning (SROS) by regional hospitalisation agencies $(\mathrm{ARH})$. More precisely, the analysis of the implementation of the SROS III clearly reveals the role of the ARH as a coordinating body, in periods of both calm and crisis, of various technical, financial and political strategies developed by regional actors. This also makes it possible to identify the outlines of a horizontal- and regional-dominant mode of regulation, reinforced by the 'threat' of vertical and national regulation, i.e. ministerial arbitration under pressure from local actors. The results of these observations are discussed with regard to models of cross-regulation and institutionalisation of collective action.This article thus highlights the local functioning of a regulatory State, that 'governs remotely' by defining frameworks for health organisation, and by leaving a certain autonomy to the ARH to implement them according to regional contexts.

(c) 2011 IDMP/Lavoisier SAS. Tous droits réservés

Keywords: hospital, health planning, regionalisation, regional health organisation plan, regional hospitalisation agency, regional health agency, health policies, heath territory, regulatory Sate, hospital system.

\section{Introduction}

\section{État, Marché et Planification hospitalière}

Les schémas régionaux d'organisation sanitaire de troisième génération (SROS III) sont indissociables des autres volets de la réforme hospitalière amorcée en 2003, c'est-àdire de l'introduction de mécanismes de régulation marchande (tarification à l'activité des établissements de santé, T2A) et du développement de mode d'organisation et de gestion issus ou inspirés du secteur privé (qualité-certification, nouvelle gouvernance). À l'image des évolutions à l'oeuvre dans le système hospitalier britannique, le renouvellement des formes d'intervention de l'État semble aller de pair avec le recours croissant à des modes d'organisation fondé sur la logique du marché (Le Galès et Scott, 2008). Plus d'État et surtout plus de règles semblent ainsi aller de pair avec plus de marché et plus de compétition (Polanyi, 1983 ; Gamble, 1994 ; Vogel, 1996 ; Le Galès et Scott, 2008). Précisément, le repositionnement et le renforcement de l'État sur des fonctions de régulation (Majone, 1997, 1999 ; Chevallier, 2004) apparaissent intimement liés avec le développement de mode d'organisation et de gestion inspiré du marché et des entreprises privées. C'est donc dans ce contexte d'interdépendance entre le repositionnement de l'État (sur un mode de gouvernement à distance) et de la montée en puissance des logiques marchandes et managériales dans le système hospitalier qu'il est possible de commencer à situer l'analyse des SROS III et du rôle des ARH.

En fait, ces analyses macro-sociologiques décrivent à la fois des tendances et des mécanismes généraux d'organisation que l'analyse micro-sociologique peut permettre de compléter ou de nuancer. « Vu d'en haut », à un niveau macro, les systèmes hospitaliers régionaux, les Agences Régionales de l'Hospitalisation (ARH) et les SROS III apparaissent de plus en plus structurés par le pouvoir normatif et régulateur de l'État, des modes d'organisation marchand et managériaux (également introduits par l'État). Vu dans d'en bas, d'un point de vue micro, l'analyse de ces systèmes, de ces agences et de ces schémas révèlent différentes configurations régionales d'acteurs, le rôle des élus et l'autonomie, relative mais réelle, des 
ARH pour articuler les points de vue techniques, financiers et politiques qui s'expriment au niveau local. Ce faisant, elle contribue à dévoiler les mécanismes et circuits de régulation ${ }^{1}$, c'est-à-dire d'ajustement des différentes stratégies d'acteurs s'exprimant dans les systèmes hospitaliers régionaux.

\section{Régulation croisée, Institutionnalisation de l'action collective et Planification hospitalière}

L'analyse de la mise en œuvre des SROS III met effectivement en évidence des mécanismes et des circuits de régulation des stratégies déployées par les différents acteurs régionaux. Rappelant la régulation croisée du système politico-administratif local antérieur à la décentralisation (Crozier et Thoenig, 1975), les mécanismes d'ajustement à l'œuvre dans la planification hospitalière semblent cependant plus proches du processus d'institutionnalisation des négociations caractérisant la gestion territoriale depuis la décentralisation (Duran et Thoenig, 1996).

La régulation croisée était complémentaire d'un mode d'administration verticale, caractéristique d'un système hiérarchique dominé par l'État. S'agissant de l'institutionnalisation des négociations, elle est associée à un mode de gestion horizontal, de systèmes locaux multi-acteurs " dont l'épicentre se situe autour du traitement territorialisé des problèmes » (Duran et Thoenig, 1996). Alors que la régulation croisée intervenait en aval des politiques publiques au travers de négociations cachées, les nouvelles formes de régulation se situent davantage en amont de l'action publique dans le cadre de négociations institutionnalisées. D'une logique quantitative de production de l'action publique fondée sur la fourniture d'équipements et de services, nous sommes en effet passés à une logique plus qualitative et préventive de résolution de problèmes, supposant la co-construction et la mise en cohérence des interventions publiques (Duran et Thoenig, 1996 ; Borgetto et Lafore, 2000 ; Michel et Bellanger, 2002).

Dans le domaine sanitaire, l'État intervient progressivement, depuis la fin de la seconde guerre mondiale, pour tenter de contrôler le fonctionnement des hôpitaux, planifier l'organisation de l'offre de soins et maîtriser les dépenses. Il s'est cependant longtemps heurté à un système hospitalier éclaté et contrôlé au niveau communal par les médecins avec le soutien des directeurs d'hôpitaux et des élus locaux (Schweyer, 1998). «Au niveau régional, la tutelle ne peut ni vraiment s'appuyer sur l'échelon central qui conclut souvent des arrangements en dehors d'elle, ni sur l'autorité préfectorale, autonome et sensible aux interpellations des élus » (Schweyer, 1998). Marquée par des arrangements cachés et des mécanismes d'ajustement entre filières administrative et politique s'apparentant à la régulation croisée, la régulation du système hospitalier a cependant évolué dans le sens de l'institutionnalisation de l'action collective et de la négociation. Dans la perspective d'une gestion plus qualitative et différenciée de l'offre de soins, les DRASS étaient déjà passées, avant la création des ARH, « d'un statut d'institution-relais du ministère de la santé chargée de l'application locale d'une politique conçue au niveau national, à celui d'une institution disposant d'une marge de manœuvre dans la définition de cette politique » (Denis et Valette,

\footnotetext{
${ }^{1}$ Le concept de régulation est cependant polysémique. Pour plus de détail, notamment sur son usage dans la littérature française, il est possible de se référer à J. Commaille et B. Jobert (1999), Les métamorphoses de la régulation politique, LGDJ, et en particulier à l'article de J.C. Thoenig, « I'usage analytique du concept de régulation ».
} 
1997). Dans ce contexte, « la régulation apparaît comme un exercice d'apprentissage où les modes de collaboration et le contenu des accords sont à développer. En imposant l'utilisation d'instrument (SROS, campagne budgétaire) dont l'actualisation reste à construire, la loi de 1991 et ses décrets accordent au local de nouvelles marges de manœuvre. L'instrument n'établit pas une relation de contrôle entre le centre et les services déconcentrés. Il pousse, volontairement ou non, à un jeu de la part des DRASS, dont l'issue et le déroulement échappent en partie à l'autorité centrale» (Denis et Valette, 1997). Entre 1990 et 1996, on observe donc « une délégation de fait, les DRASS se construisant pas à pas en un espace stratégique de régulation même si le dispositif formel les considère comme des courroies de transmission, des traducteurs de règles nationales. Avec la création des ARH, cette délégation s'est institutionnalisée » (Fargeon et al., 2002).

À la veille du passage aux agences régionales de santé (ARS) et après 13 années de fonctionnement des ARH, depuis le passage progressif à la T2A et l'intensification des appels au développement des coopérations, nous nous sommes interrogés sur l'évolution du processus de planification hospitalière ainsi que sur la nature et la portée du travail assuré par les $\mathrm{ARH}^{2}$. Comment les ARH ont-elles mis en œuvre le dispositif de planification conçu par l'État pour des établissements de santé de plus en plus financés par la T2A, gérés sur le modèle des entreprises privées et appelés à coopérer avec les autres acteurs de santé ? Quels sont précisément les formes et les fonctions de la partie déconcentrée, d'un État central régulateur, qui « gouverne à distance » en définissant les cadres de l'organisation de l'offre de soins, en laissant aux ARH le soin de les mettre en œuvre en fonction des contextes régionaux?

\section{ARS, ARH et SROS III}

La création des Agences Régionales de Santé (ARS) est intervenue le $1^{\text {er }}$ avril 2010 , avec comme leitmotiv le décloisonnement et le développement des coordinations entre l'hôpital et la médecine de ville, le sanitaire et le médico-social, les soins hospitaliers et la santé publique... Elles sont censées poursuivre et prolonger le chemin tracé depuis 1996 par les Agences Régionales de l'Hospitalisation (ARH) dans le domaine hospitalier.

Dans un contexte de rationalisation des dépenses de santé, les ARH ont effectivement été créées en 1996 pour assurer une meilleure coordination du système hospitalier, entre les établissements de santé publics et privés, entre les services de l'État et de l'Assurance maladie. Elles ont également été créées pour éviter ou limiter les interférences politiques dans la gestion de l'organisation de l'offre de soins.

La concurrence entre les établissements de santé publics et privés, associée à la séparation entre les services de l'État et de l'Assurance maladie, ainsi que la perméabilité de ces services aux comportements stratégiques des établissements relayés par les élus locaux, étaient régulièrement présentés comme les facteurs explicatifs des difficultés de la planification du système hospitalier, de la restructuration des établissements et du développement des complémentarités entre le public et le privé (Denis et al., 2001). « Par la personnification de la fonction de régulation, la spécialisation du champ de compétences et la plus grande autorité dont dispose le directeur d'ARH, autorité tirée de son statut de représentant de

\footnotetext{
${ }^{2}$ Une sollicitation et le soutien financier de l'Association des régions de France (ARF) nous ont permis de mener à bien cette recherche.
} 
l'État chargé de faire appliquer la politique de l'État en matière hospitalière, les ARH créent un niveau intermédiaire de l'État, susceptible d'être plus robuste face à la pression des élus locaux et des établissements et de limiter les inefficacités liées à la gestion d'intérêts contradictoires (restructurations hospitalières et aménagement du territoire ou maintien de l'emploi » (Denis et al., 2001). C'est aussi pour cette raison que la régionalisation ne s'est pas accompagnée de la décentralisation politique. La gestion du système hospitalier est « confiée à une agence et non aux conseils régionaux, considérés comme étant trop vulnérables aux pressions des élus politiques locaux » (Kerleau, 2003).

Ainsi « dépolitisées », les ARH sont conçues sur le modèle de l'agence. Elles constituent « le premier exemple d'application de la 'technique agencielle' à la politique déconcentrée des pouvoirs publics jusqu'alors réservée à l'échelon central » (Maquart, 1996). Pour assurer le pilotage du système hospitalier, les ARH, dotées d'un statut de groupement d'intérêt public (GIP), sont conçues comme des administrations de missions très personnalisées, ce qui suscite des débats interministériels très importants avec le ministère de l'intérieur, dans la mesure où la création des ARH entraîne la dépossession des préfets de leurs attributions hospitalières (Denis et al., 2001). Afin d'asseoir la légitimité des ARH au plan local, il est ainsi décidé que les directeurs d'ARH seront nommés en conseil des ministres, à l'instar des Trésoriers Payeurs Généraux, des préfets et des recteurs d'académie. À l'aide des services de l'État et de l'Assurance maladie qui exerçaient précédemment ses missions ${ }^{3}$, les ARH sont chargées de définir et de mettre en œuvre la politique régionale d'offres de soins hospitaliers, d'analyser et de coordonner l'activité des établissements de santé publics et privés et de déterminer leurs ressources. Elles ont ainsi vocation à réguler le système hospitalier, au travers de l'allocation des ressources, de la planification sanitaire et de la contractualisation avec les établissements.

Amorcée en 2003, la modernisation du système hospitalier intervient dans une perspective globale (Michel et Bellanger, 2002). Avec le passage progressif à la T2A, les fonctions d'allocation des ressources des ARH se réduisent. Un processus de recentrage des agences sur les fonctions de coordination des acteurs et des activités sanitaires se dessine dans le cadre notamment des SROS 3. Avec la T2A, le financement des établissements n'est plus fondé sur un budget global reposant sur une enveloppe de dépenses revalorisée chaque année et allouée par les ARH, mais sur les recettes effectivement dégagées par les activités. La T2A incite en principe les établissements de santé à se positionner sur les segments d'activité rentables sur un territoire donné. Elle milite pour un positionnement stratégique, une spécialisation des établissements sur leur territoire afin de conserver ou de conquérir des parts de marchés. En basant le financement sur les recettes dégagées par chaque établissement sur un marché concurrentiel, l'État introduit un mécanisme extrêmement puissant de régulation du système hospitalier par le marché. Ce modèle de gestion fait appel aux outils de l'analyse stratégique popularisés par la matrice d'analyse croisant les forces et faiblesses d'une organisation avec ses opportunités et menaces. En complément de la possible régulation par les tarifs au niveau national, les SROS ont vocation à amortir les effets négatifs de la T2A (Tonneau et Moisdon, 2008).

\footnotetext{
${ }^{3}$ Les ARH se voient en effet confier les missions jusqu'alors exercées par les DRASS, les DDASS, les préfets de régions et de départements en qui concerne les établissements de santé publics ; elles se voient également confier les missions exercées par les Caisses Régionales d'Assurances Maladies (CRAM) pour ce qui relève des établissements privés.
} 
D'un point de vue technique, les SROS 3 ont pour objectif de prendre en compte les besoins de santé et de soins de la population et de les décliner dans des objectifs de santé, eux-mêmes déclinés en objectifs quantifiés permettant d'atteindre les résultats attendus. Ces objectifs comprennent une borne haute d'activités à ne pas dépasser et une borne basse d'activités à atteindre, par territoires de santé (OQOS) et par établissements (OQE). Ces orientations et ces objectifs régionaux doivent être en principe alimentés par des préprojets médicaux de territoire puis déclinés par des projets médicaux de territoire (PMT), élaborés par des conférences sanitaires de territoires (CST). Ces dernières sont composées non seulement de professionnels de santé (hospitaliers et libéraux, établissements publics et privés) mais aussi d'élus et d'usagers.

Réduites en matière d'allocation des ressources depuis le passage à la T2A, les fonctions des ARH se recentrent sur la planification des activités hospitalières et la coordination des différents acteurs (établissements de santé publics et privés, professionnels libéraux, élus, usagers...).

Pour remplir ces missions, les agences sont conduites à articuler, dans le calme ou dans la crise, deux types d'approches, techniques et politiques :

- d'un côté, des démarches techniques, planificatrice et /ou gestionnaire, fondée sur les démarches projet, l'analyse démo-géographique, les normes de qualité, de sécurité et les exigences de maîtrise des dépenses (Jourdain et Bréchat, 2008);

- de l'autre, des approches politiques destinées à défendre ou promouvoir des intérêts locaux et/ou corporatistes.

Cette tension entre les logiques techniques et politiques constitue le fil directeur de cet article traitant du processus de planification de l'offre de soins dont la régulation à dominante horizontale, comporte à la fois des points communs et des différences avec les modèles de la régulation croisé et de l'institutionnalisation de l'action collective.

\section{Données utilisées}

Une analyse des SROS 3 permet de préciser la nature du travail réalisé par les ARH, ainsi que les mécanismes et circuits de régulation à l'œuvre dans le système hospitalier. Cette analyse est basée sur les résultats d'un bilan national réalisé au cours de l'année 2007 à partir de l'analyse documentaire de l'ensemble des SROS arrêtés au 31 mars 2006, complétée par des entretiens avec des représentants des ARH dans les 22 régions métropolitaines. Cette recherche a ensuite été approfondie par une analyse du processus de régionalisation dans 6 régions métropolitaines sélectionnées après une analyse factorielle des correspondances (AFC) croisant des indicateurs territoriaux (existence ou non de processus PMT), contractuels (niveau de contractualisation), politiques (existence ou non d'une opposition ayant bloqué l'élaboration du SROS), démographiques et sanitaires.

Ces 6 régions illustrent les différentes combinaisons possibles des deux principaux paramètres mis en évidence par l'analyse factorielle : l'offre de services (densité médicale, lits par habitants) et la démographie (âge et densité de population). Les exemples du NordPas-de Calais, Poitou-Charentes et de la Haute-Normandie (cf. annexe 1) sont largement présentés dans la deuxième partie de cet article. Ces trois régions se caractérisent par une faible offre de soins, à laquelle s'ajoute une forte surmortalité pour la première, un fort degré de vieillissement pour la seconde, la dernière étant dans une situation intermédiaire. Parmi ces trois régions, Nord-Pas-de-Calais et Poitou-Charentes ont vécu un conflit majeur lors de l'élaboration de leur SROS. 
Globalement, cet article se concentre sur la manière dont la tension entre approches techniques et politiques imprègne l'organisation territoriale infra-régionale $(\$ 1$.) et plus généralement les différents modes d'élaboration des SROS III par les ARH (§ 2.).

\section{L'organisation territoriale infra-régionale}

Dès 2004, le ministère de la santé insiste sur la nécessité d'instaurer une dynamique territoriale. «Les SROS de troisième génération se déclineront autour de quatre priorités : une meilleure évaluation des besoins de santé, une plus grande prise en compte de la dimension territoriale, une association plus étroite des établissements, des professionnels, des élus et des usagers, une véritable animation de leur mise en oeuvre au sein de chaque territoire de santé ${ }^{4} »$.

S'agissant de l'évaluation des besoins de santé, l'ensemble des SROS y fait référence. Pourtant, il n'y a guère de définition des notions de besoins de soins, de besoins de santé et des démarches permettant de les analyser. En fait la plupart des SROS semble fonder leur démarche sur la consommation et la production des soins. En ce sens, les SROS sont organisés autour de l'activité des établissements de santé et relèvent d'approches « hospitalo-centrées ».

Au niveau territorial, de nombreuses différences d'organisation apparaissent notamment dans les méthodes de construction des territoires de santé et surtout dans les systèmes de gradation des soins mis en place au niveau infra-régional qui ne seront cependant pas détaillés dans le cadre de cet article. La priorité est ici donnée à l'analyse des modes de construction (I) et d'animation (II) des territoires de santé de recours où les tensions entre les approches techniques et politiques sont particulièrement présentes.

\subsection{La construction des territoires de santé de recours : la combinaison variable des logiques techniques et politico-administratives}

Plus ou moins rationalisés au fil des différentes réformes (256 à l'origine en 1974, 223 en 1984, 152 en 1994 et 143 en 2004), les secteurs sanitaires institués par la loi hospitalière de 1970 restaient encore souvent attachés aux frontières départementale et/ou régionale. Pour le passage des secteurs sanitaires aux territoires de santé, le ministère recommande en 2004 de construire les nouveaux territoires sur la base de zones d'attraction théorique et réelle ou encore de zones à risque ${ }^{5}$, « indépendamment des limites administratives (départements ou régions) ${ }^{6} \gg$.

En pratique, la construction des territoires représente un premier exemple de tension existant entre l'approche technique, faisant usage des outils de la géographie de la santé, et

\footnotetext{
${ }^{4}$ Circulaire du 5 mars 2004 relative à l'élaboration des SROS de troisième génération.

${ }^{5}$ L'annexe 2 de la circulaire du 4 mars 2004 fait l'inventaire des différentes méthodes techniques de zonage en distinguant celles conduisant à la délimitation de zone d'attraction théorique (fondée sur la géométrie : courbe isochrone, polygone de Thiessen et aire de Reilly) ou réelle (basées sur l'analyse des flux entre le domicile et le lieu de soin, ou le domicile et le lieu de travail ou de recours à des services et équipements : bassin hospitalier, bassin de médecine générale, bassin de soins ambulatoires, bassin de vie ou villes et bourgs attractifs de I'INSEE, zone d'emploi, zonage en aires urbaines et en aires d'emploi de l'espace rural, zonage IRIS à l'intérieur des villes) et celles visant à identifier des zones à risques ou défavorisées.

${ }^{6}$ Circulaire ${ }^{\circ}$ 101/DHOS/O/2004 du 5 mars 2004 relative à l'élaboration des SROS de troisième génération, p. 3
} 
l'approche politique accordant davantage d'importance aux découpages administratifs ou électoraux du département et de la région. Le mode de construction des territoires de santé de recours peut être lu en fonction des critères techniques explicites de définition des territoires, puis des critères politico-administratifs implicites qui transparaissent dans la correspondance entre les périmètres des territoires de santé et les frontières départementales et régionales.

\subsubsection{La diffusion des critères technique de construction}

Les critères techniques, c'est-à-dire les modes de déplacement et de vie de la population, apparaissent assez largement dans les méthodes de construction des territoires de santé utilisées dans les régions (Coldefy et Lucas-Gabrielli, 2008).

Les deux tiers des régions métropolitaines ont effectivement procédé au redécoupage des territoires de santé de recours en fonction des déplacements de la population vers des structures de soins ou vers d'autres types de services. 11 régions ont pris en compte les flux de fréquentation hospitalière (6 régions) en les articulant avec les bassins de vie ( 5 régions). D'autres ont intégré dans leur démarche les zones d'attraction hospitalière et ambulatoire (3 régions). Enfin, seule une région (Nord-pas-de-Calais) s'est exclusivement basée sur les bassins de vie.

Toutefois, «malgré la consigne 'd'emprunter une voie innovante' pour ces découpages, un tiers des régions a fait le choix de maintenir de manière totale ou partielle le découpage des secteurs sanitaires précédemment mis en place dans le SROS II » (Coldefy et LucasGabrielli, 2008). Parmi ces dernières, certaines avaient cependant retravaillé, suivant des approches techniques, la carte de leur secteur sanitaire dans le cadre des SROS précédents.

Nuançant la montée en puissance de la « technicisation » des territoires, l'examen du périmètre des territoires de santé de recours montre l'importance persistante des cadres départementaux et régionaux. Bien que ces cadres correspondent plus ou moins aux espaces de déplacement et de vie de la population, ils traduisent aussi une structuration politicoadministrative des territoires de santé.

En fait, les critères techniques ont été combinés de façon variable selon les régions avec des critères politiques.

\subsubsection{La persistance d'une structuration départementale}

Rappelant l'étendue de la technicisation, l'analyse comparée des périmètres des territoires de santé et des départements révèle aussi la persistance du prisme départemental :

- 12 régions se sont apparemment émancipées des cadres départementaux pour définir des territoires de santé de recours suivant des logiques plus techniques. Toutefois, à l'exemple de la Haute-Normandie, l'émancipation par rapport au cadre départemental ne signifie pas l'imperméabilité aux influences politiques locales (cf. annexe).

- Dans les 10 autres régions métropolitaines, le périmètre départemental apparaît (complètement ou partiellement, strictement ou par regroupement) dans la carte des territoires de santé. Tout d'abord, 17 territoires de santé de recours ${ }^{7}$ sont strictement départementaux.

\footnotetext{
${ }^{7}$ Les 6 territoires de santé de la région Centre. Les 3 territoires de santé du Limousin. En Midi-Pyrénées, les 5 territoires de santé du Lot, du Tarn et Garonne, du Gers, des Hautes Pyrénées, de l'Ariège. En LanguedocRoussillon, celui des Pyrénées-Orientales. En Bourgogne, le territoire de la Côtes d'Or.
} 
Ensuite, par agrégation, de multiples territoires de santé infra-départementaux ${ }^{8}$ respectent aussi parfaitement les frontières de 8 autres départements. Enfin, l'empreinte départementale est également manifeste dans de nombreux autres territoires de santé dessinés sur un patron départemental aménagé à la marge ${ }^{9}: 11$ départements auxquels sont ajoutés ou soustraits quelques cantons constituent la base de plusieurs territoires de santé.

Au total, ce sont donc 36 départements, soit plus d'un département sur trois, qui façonnent plus ou moins, mais indéniablement, le périmètre des territoires de santé de recours. Selon les départements, certains conseils généraux, avec leurs élus, et/ou certaines administrations départementales telles que les DDASS, continuent ainsi à résister au développement de la technicisation des territoires sanitaires.

\subsubsection{Le respect systématique des frontières régionales}

Entre logique technique et logique politico-administrative départementale, la construction des territoires de santé de recours est aussi structurée par des logiques régionales. La comparaison des territoires de santé aux frontières régionales est particulièrement éclairante. L'ensemble des territoires de santé de recours respecte scrupuleusement les limites administratives régionales. Il n'y a aucun exemple de territoire de santé de recours débordant les frontières régionales. Pourtant, les exemples de communes ou cantons limitrophes soumis à l'attraction d'un territoire de santé d'une autre région sont multiples ${ }^{10}$. Autrement dit, bien que les flux sanitaires dépassent dans de nombreux cas les limites régionales, le périmètre des territoires de santé de recours s'arrête systématiquement à la frontière de chaque région.

Des coopérations entre régions sont bien prévues dans le cadre de schémas interrégionaux (SIOS), mais ceux-ci concernent simplement quelques domaines d'activités extrêmement spécialisés. Pour ce qui est des soins plus ordinaires proposés à l'échelle des territoires de recours, la population n'hésite pas dans les configurations limitrophes à dépasser les frontières régionales qui restent cependant infranchissables pour les institutions régionales. À cet égard, il aurait été intéressant d'identifier plus précisément les acteurs (les ARH, les DRASS, les services de l'Assurance Maladie, ou encore les conseils régionaux, voire les conseils généraux...) qui sont à l'origine de cet immobilisme régional.

\subsubsection{Des ajustements à la marge sur des territoires politiques de projets}

Alors qu'elles tendent à s'opposer dans la construction des territoires de santé, les approches techniques et politico-administratives peuvent dans certains cas converger. Un

\footnotetext{
${ }^{8}$ Les deux territoires de santé de l'Aveyron, du Tarn, de la Saône et Loire ; les 6 territoires de santé de la Haute-Garonne. Les 3 territoires de santé du Val d'Oise, des Yvelines, des Hauts-de-Seine, de Paris.

${ }^{9}$ Les territoires de santé de la Lozère, du Lot-et-Garonne, de la Corse, de la Vienne, des Deux-Sèvres, de la Charente-Maritime, de la Charente, de la Mayenne, la Gironde, le Périgord, la Nièvre,

${ }^{10}$ Gisors (Haute-Normandie) à la limite de l'llle de France ; les cantons du nord de la Mayenne (Pays de la Loire) sous l'attraction d'Alençon (Basse Normandie); certains cantons du nord de la Loire-Atlantique (Pays de la Loire) par rapport à Redon (Bretagne). Les cantons du nord des Deux-Sèvres (Poitou-Charentes) par rapport à Cholet (Pays de la Loire) ; le sud Indre et Loire (Centre) et Châtellerault (Poitou-Charentes) ; les cantons au sud de Laon (Picardie) sous l'influence de Reims (Champagne-Ardenne) ; le territoire du Puy-enVelay (Auvergne) par rapport à Saint-Etienne (Rhône-Alpes) ; l'ouest du Gers (Midi-Pyrénées) par rapport à Mont-de-Marsan (Aquitaine) ; le sud de la Charente-Maritime (Poitou-Charentes) et Libourne (Aquitaine)...
} 
important travail d'ajustement des territoires de santé sur les territoires intercommunaux a été ainsi réalisé dans la région PACA. Le périmètre des 9 territoires de santé de recours subdivisés en 25 territoires de proximité s'imbriquent dans la cartographie de l'intercommunalité (Pays, communauté d'agglomération, communautés urbaines et communauté de communes). De cette façon, les zones dessinées par les flux hospitaliers sont positivement articulées avec la dynamique des «nouveaux » territoires politiques locaux.

La construction des territoires de santé de recours révèle une «tectonique des plaques ». D'un côté, un mouvement de technicisation visant à adapter l'organisation sanitaire au mode de déplacement et de vie de la population, se développe et monte en puissance. De l'autre côté, les cadres départementaux résistent dans plus d'un tiers des départements et surtout les frontières régionales apparaissent infranchissables d'un point de vue institutionnel. À la marge, les espaces intercommunaux forment le territoire d'entente entre approches techniques et politiques.

Après avoir mis en évidence les logiques techniques plutôt «innovantes » et les logiques politico-administratives départementales et régionales plutôt « conservatrices » qui façonnent la construction et les périmètres des territoires de santé, on peut s'interroger sur la nature et les modalités des animations à l'œuvre dans ces territoires de santé.

\subsection{L'animation territoriale : I'impulsion des Agences Régionales de l'Hospitalisation et le contrôle de la Fédération Hospitalière de France (FHF)}

Sur les nouveaux territoires de santé, des conférences sanitaires de territoire (CST), présidées par un élu et rassemblant la diversité des acteurs locaux concernés par la santé, ont vocation à élaborer un projet médical de territoire (PMT). En principe fondés sur un diagnostic local et destinés à promouvoir la coopération entre établissements, la mise en place de filières et de réseaux, les PMT sont censés, d'une part, préparer l'élaboration du schéma régional d'organisation sanitaire (ils ont été appelés pré-PMT) et, d'autre part, une fois le SROS arrêté, servir à sa mise en œuvre au niveau de chaque territoire de santé (PMT). L'idée de ces projets de territoire est de conduire les établissements à raisonner non plus directement au niveau de leur établissement mais d'abord au niveau du territoire de santé pour saisir les besoins de santé de la population, et définir la place de chaque établissement dans la production de l'offre de soins nécessaires sur ce territoire. En ce sens, le SROS et sa déclinaison territoriale visent à amortir les effets du passage du financement des hôpitaux à la tarification à l'activité et celui de la compétition entre les établissements de santé.

En fait, comment les différents acteurs locaux se sont-ils saisis de ce dispositif ou s'y sont-ils inscrits ?

\subsubsection{Une région sur deux engagée dans la démarche PMT}

Le bilan national des SROS 3 que nous avons réalisé révèle tout d'abord une très grande disparité dans la mise en œuvre des projets médicaux de territoire (PMT). Plus d'un an et demi après la promulgation des SROS 3, soit à la fin de l'année 2007, seule la moitié des 22 régions métropolitaines était engagée dans une démarche d'animation territoriale.

Au moment de l'enquête, dans 11 régions, la démarche de projet médical de territoire est absente (pas de pré-PMT, ni de PMT) ou incomplète (quelques PMT mais pas de pré- 
PMT). 5 régions se caractérisent ainsi par une absence complète de pré-PMT et de PMT : l'Ille de France, la Haute-Normandie, la Champagne-Ardenne, la Corse et le Nord-Pasde-Calais (où quelques PMT étaient cependant en voie de formalisation). Les 7 régions qualifiées d'incomplètes se caractérisent par une absence de pré-PMT et un niveau réduit ou incomplet de production des PMT : Midi-Pyrénées (2 PMT réalisés sur 15), PoitouCharentes (3 PMT sur 5), Centre (4 sur 6), Auvergne ( 7 sur 9), Basse-Normandie (4 sur 5). Le Languedoc Roussillon et l'Aquitaine ont aussi été rattachés à ce groupe dans la mesure où en dépit d'une couverture complète des territoires de santé par des PMT, il n'y a eu, en revanche, aucun pré-PMT dans ces régions.

Inversement, dans les 11 autres régions (Picardie, Pays de la Loire, Franche-Comté, Rhône-Alpes, Bourgogne, Limousin, Alsace, Lorraine, PACA, Bretagne), l'animation territoriale a démarré en amont du SROS par la réalisation de pré-PMT et s'est poursuivie ensuite par la production de PMT dans la totalité ou la majeure partie des CST. Par exemple, en Rhône-Alpes, les 13 pré-PMT se sont transformés en 13 PMT. En Picardie, les CST ont non seulement produit des pré-PMT, mais également une première puis une seconde génération de PMT.

\subsubsection{Des projets médicaux de territoire dépendant de la stratégie territoriale des ARH}

En fait, le niveau d'avancement de la démarche PMT est étroitement lié à la stratégie territoriale des ARH. Dans les régions où le processus PMT a fonctionné, en se traduisant par des pré-PMT puis par une première vague de PMT voire une seconde, les agences se sont effectivement mobilisées pour encadrer et/ou accompagner le fonctionnement des conférences sanitaires de territoire (CST).

Selon les ARH, les moyens mis à la disposition des acteurs locaux sont financiers (financement du recrutement d'un chargé de mission pour l'animation des conférences et la réalisation des PMT), matériels (mise à disposition de locaux et/ou d'information pour la réalisation des diagnostics territoriaux), méthodologiques (lettre de cadrage décrivant la méthode et/ou les thèmes à travailler). Par exemple, dans les Pays de la Loire, l'ARH a financé le recrutement d'un chargé d'étude pour la réalisation de chaque PMT. Elle a laissé les conférences de territoire libres de traiter les thèmes de leur choix dans le cadre des pré-PMT ; mais elle a ensuite cadré la réalisation de la première vague de PMT en leur demandant de travailler sur des thèmes précis. Elle a ensuite reconduit le financement des chargés d'études pour la réalisation d'une deuxième vague de projets en fonction de nouveaux thèmes également définis par l'ARH. Autre exemple : en Champagne-Ardenne, bien que l'ARH n'ait pas apporté de soutien financier au fonctionnement des conférences, elle les a mobilisées par la mise en place d'un système d'animation locale. En lien avec des référents de l'agence, des acteurs de terrain d'origine diverse (directeur d'établissement privé, directeur d'hôpital public, présidents de CME) ont fait office d'animateurs territoriaux et ont permis la réalisation de pré-PMT puis de PMT sur chaque territoire de santé.

Inversement, dans les régions où il n'y a pas eu de pré-PMT et finalement assez peu de PMT, il n'y a pas eu non plus de mobilisation des ARH en faveur d'une animation territoriale. Dans la mesure où les projets médicaux de territoire n'ont pas semblé constituer une priorité pour ces ARH, les agences n'ont pas déployé de moyens financiers, matériels ou méthodologiques pour structurer et dynamiser le fonctionnement des conférences ; elles 
n'ont pas non plus cherché à susciter la mise en place de système d'animation par les acteurs locaux. Dans ces régions où l'animation territoriale n'apparaît pas prioritaire, l'absence d'implication et d'impulsion par les ARH se traduit par une absence de pré-PMT et une absence ou un nombre très réduit de PMT.

La réalisation des projets médicaux de territoire dépend ainsi de la stratégie territoriale des ARH. Dès lors qu'ils sont livrés à eux-mêmes, les différents acteurs des conférences sanitaires de territoire ne semblent pas en mesure de se coordonner pour réaliser un diagnostic et un projet partagés. Au contraire, si les ARH se mobilisent, les conférences s'engagent dans un travail, au moins formel, de production de pré-PMT et de PMT.

Pour mieux cerner la portée de l'animation territoriale, il apparaît utile de s'interroger sur le contenu des projets médicaux de territoire. Les différents acteurs se sont-ils positionnés et coordonnés au niveau du territoire de santé ou ont-ils simplement continué à défendre les intérêts de leurs établissements ou de leur profession? Il apparaît également utile de réfléchir sur les conditions d'installation et de fonctionnement des conférences sanitaires de territoire (CST). Ont-elles fonctionné sur un mode pluraliste ou ont-elles été contrôlées ou influencées par certains acteurs et selon quelle logique ? Quelques tendances sur l'organisation interne des conférences et le contenu des PMT se dégagent effectivement à partir des 6 enquêtes qualitatives réalisées dans les régions ? Le fonctionnement des CST parait structuré par des logiques professionnelles corporatistes et des logiques politiques.

\subsubsection{Des conférences sanitaires de territoires sous le contrôle de la Fédération Hospitalière de France}

Dans les 6 régions enquêtées, ce sont bien des directeurs d'hôpitaux publics qui assurent le fonctionnement administratif des conférences sanitaires de territoire. La Fédération Hospitalière de France (FHF) semble s'être saisie directement ou indirectement des fonctions de secrétariat de ces conférences.

Par exemple, dans l'une des régions enquêtées,

"les CST se sont mises en place tardivement, mais depuis qu'elles se sont mises en place, elles travaillent. La FHF n'y est pas pour rien, car nous avons réussi à imposer comme secrétaire des 4 conférences un patron de la FHF. Chaque CST a comme secrétaire un représentant de la FHF. On peut par ce biais donner des consignes générales dans les conférences. » (représentant de la FHF)

Cette prise de contrôle de la FHF a d'ailleurs suscité certaines réactions de la part de la Fédération de l'Hospitalisation Privée (FHP).

"Sur le territoire du H., on a vu l'hôpital de V. s'emparer du secrétariat et rédi-

ger les procès-verbaux. Nous nous sommes opposés à certains procès-verbaux...» (représentant de la FHP)

Dans une autre région, le représentant de la Fédération Hospitalière Privée indique que « sur les 5 territoires de santé, le secrétariat a été assuré par le public, mais cela n'a pas posé de problème, leur influence a été limitée $»^{11}$. Un représentant de la Fédération Hospitalière de France précise d'ailleurs que « la FHF a hérité du secrétariat des CST sur recomman-

${ }^{11}$ Entretien avec un représentant de la FHP 
dations de $\mathrm{l}^{\prime} \mathrm{ARH}{ }^{12}$ ». À l'exemple de ces deux régions où les représentants de fédérations se sont clairement exprimés, la FHF s'est emparée ou a été investie de la responsabilité du secrétariat des conférences sanitaires de territoire.

De la même façon, en Alsace et en Haute-Normandie, ce sont les 4 directeurs des centres hospitaliers de référence qui pilotent les conférences. En Aquitaine, le secrétariat des CST est également assuré par des directeurs d'établissements publics. Enfin, en Auvergne, seule région à avoir opté pour un mode d'organisation territorial ad hoc pour animer des «projets territoriaux de santé », sans président élu, ni secrétaire, les projets médicaux de territoires sont élaborés par un coordinateur choisi au sein d'un groupe de travail, parmi les directeurs d'établissements publics voire présidents de commission médicale d'établissement, épaulé pour l'organisation matérielle par un inspecteur de la DDASS. Cette «emprise » de la Fédération hospitalière de France et des hôpitaux publics sur le secrétariat des conférences et la réalisation des PMT permet aux hôpitaux publics, selon les régions, d'orienter, de contrôler ou de superviser le fonctionnement des conférences et le contenu des PMT. Si des logiques professionnelles corporatistes sont ainsi à l'œuvre dans les conférences, des logiques d'actions politiques s'expriment également par l'intermédiaire, notamment, des présidents de conférence sanitaire de territoire qui sont depuis 2005 obligatoirement des élus.

\subsubsection{L'amorce de l'institutionnalisation du rôle des élus dans les Conférences Sanitaires de Territoire}

Différentes conceptions politiques de l'organisation de l'offre de soins émergent peu à peu. Les élus interviennent souvent dans le cadre des conférences (CST), mais également au niveau de l'ARH ou du ministère, pour défendre les emplois et une offre de soins de proximité menacés par des restructurations. Avec certains présidents de conférence sanitaire de territoire, une approche en termes d'aménagement des territoires de santé, fondée sur l'égalité d'accès à l'offre de soins au niveau infra-régional, commence à se préciser. Toutefois, les élus ne semblent pas encore s'être vraiment appropriés la question hospitalière. Soit ils réagissent en défendant les établissements de santé publics et privés menacés ou en soutenant leur projet de développement, soit ils prolongent au niveau des territoires de santé infra-régionaux ou régionaux ${ }^{13}$ les logiques techniques de qualité et de sécurité menant à la spécialisation et à la concentration de l'offre de soins.

L'analyse du fonctionnement des conférences est particulièrement instructive. Par exemple, tout en rappelant le rôle structurant de la FHF et /ou des professionnels de santé dans les décisions et l'activité des conférences, les présidents de conférences montrent bien la diversité du rôle des élus. Sur l'un des territoires de santé de la région Poitou-Charentes,

\footnotetext{
12 Entretien avec un représentant de la FHF.

${ }^{13}$ Le Conseil régional du Nord-Pas-de-Calais participe à titre expérimental depuis 2007 au pilotage du système de santé régional. II participe à hauteur de 48 millions d'euros essentiellement au financement d'équipements hospitaliers. En contrepartie, six élus régionaux participent avec voix délibérative à parité avec les représentants de l'Etat et de l'Assurance maladie, à la commission exécutive de l'ARH. Dans ce contexte, certains conseillers régionaux militent pour une démocratisation du système hospitalier par l'intégration des élus au processus de décision, pour une articulation du soin à la prévention, pour le maillage du territoire par un système hospitalier spécialisé relié à des maisons de santé.
} 
« la CST est portée par le directeur du CH de M. , avec le soutien du directeur du CH de $N$., la participation des présidents de commission médicale d'établissement et certains membres du secteur privé. Les élus font valoir leurs problèmes locaux ou alors ils sont absents. Ils défendent leur propre territoire notamment du fait de la multiplication par cinq de la population pendant l'été...» (président de conférence et ancien préfet).

Sur un autre territoire de santé, les stratégies de la diversité des acteurs politiques régionaux, mais aussi de quelques acteurs techniques, apparaissent encore plus clairement.

"Les présidents de CST se sont rencontrés par le biais de la FHF. Au début, la FHF a beaucoup contribué au fonctionnement de la CST. Les maires des villes du département se sont impliqués. Au niveau de la réflexion, ce sont les médecins qui ont fait comprendre aux élus qu'un territoire de santé unique était indispensable. Il y a eu une prise de conscience des élus. Les médecins et les directeurs d'hôpitaux ont dit qu'il n'y avait pas 36 solutions » (président de CST, conseiller général).

Toutefois, dans cette région où le Conseil régional militait pour la création d'un sixième territoire de santé par la subdivision d'un territoire de santé de dimension départementale, le président de la conférence concerné, professionnel de santé et également conseiller général, s'est prononcé en faveur du maintien d'un seul territoire de santé sur cette zone. Il n'est pas simplement intervenu en relais conforme de l'avis des experts ; il s'est activement opposé à la création de ce sixième territoire, en refusant par exemple l'entrée de représentants d'un collectif en faveur de la scission territoriale au sein de la conférence.

\subsubsection{Des Projets Médicaux de Territoire (PMT) entre logiques individualiste et collective}

Le contenu des PMT semble osciller entre logiques individualiste et collective. À ce sujet, l'analyse de l'ARH Poitou-Charentes est particulièrement éclairante. Dans cette région, il n'y a pas eu de pré-PMT ; en ce qui concerne la réalisation des projets médicaux de territoire, l'ARH a mandaté les Directions départementales des affaires sanitaires et sociales pour piloter le dispositif et réaliser des diagnostics sur chaque territoire, avec l'appui de l'Union régionale des caisses d'assurance maladie. En août 2007, 3 PMT sur 5 sont formalisés. Parmi, ces trois PMT, deux d'entre eux relèvent plutôt de logiques individualistes « de type catalogue, avec le constat de manques et la formulation de demandes » et un autre d'une logique plus collective, «dans la dynamique du SROS ${ }^{14}$. Autrement l'existence d'un PMT ne signifie pas forcément qu'un projet collectif existe sur le territoire. Les projets « individualistes » reposent sur la somme de demandes individuelles émanant des établissements alors que les PMT « collectifs » sont basés sur la réflexion des établissements à l'échelle du territoire de santé et en fonction des orientations du SROS, permettant ainsi de dégager des axes de coopérations, la mise en place de filières ou de réseaux.

\subsubsection{Des dynamiques territoriales variables selon le degré de concurrence des thématiques, la taille et la nature des établissements concernés}

Dans le contexte de la tarification à l'activité des hôpitaux, les dynamiques peuvent aussi varier selon les thèmes abordés dans les PMT. Par exemple, en Nord-Pas-de-

\footnotetext{
${ }^{14}$ Entretien avec un cadre de l'ARH Poitou-Charentes.
} 
Calais, le président d'une conférence souligne les différences de fonctionnement selon les groupes de travail.

"On a des commissions de travail qui fonctionnent plus ou moins bien selon les sujets. Sur les personnes âgées, ça va. Sur la cancérologie qui comporte des actes techniques, il y a une concurrence très forte... On a un état des lieux et on va passer à une deuxième étape sur les modalités de prise en charge. Sur les urgences, ça a été un peu compliqué au démarrage » (président de CST).

L'ARH constate aussi les différences de fonctionnement des conférences compte tenu notamment des difficultés des fédérations, FHF, FHP et FEHAP à s'entendre sur certains territoires. Le rayonnement régional de certains établissements tels que le CHU ou des grandes cliniques ne facilite pas non plus, selon l'ARH, le sentiment d'appartenance à un territoire et le développement des coopérations ${ }^{15}$. L'ensemble de ces questions mériterait évidemment des compléments de recherche.

En conclusion, au niveau territorial, les logiques techniques et politiques se combinent de façon variable pour la construction des territoires de santé, révélant la persistance des structures départementale et régionale dans les systèmes hospitaliers régionaux. S'agissant de l'animation des territoires, le fonctionnement des conférences (CST) est manifestement sous le contrôle ou l'influence des établissements de santé publics et de la FHF. Toutefois l'élaboration des pré-PMT et des PMT dépend complètement de la stratégie territoriale des ARH. L'absence d'impulsion de l'ARH se traduit par une inertie des conférences. Au contraire, l'engagement de l'ARH, par le déploiement de moyens financiers, matériels ou méthodologiques au service des conférences, entraîne la mobilisation, au moins formelle, des acteurs locaux pour la réalisation de pré-PMT et/ ou de PMT. Les élus, quant à eux peuvent prolonger le travail des professionnels de santé, participer à l'élaboration du projet et le relayer vers l'ARH, mais ils peuvent tout autant bloquer ce travail quand ils se situent en défenseurs d'intérêts locaux.

A la fin de l'année 2007, seule une région sur deux est engagée dans une démarche d'animation territoriale. Et la coordination effective des établissements et des professionnels de santé semble encore très variable et dépendante, dans un contexte concurrentiel, des thématiques traitées dans les PMT et de la configuration de l'offre de soins sur ces territoires.

Au niveau infra-régional, les systèmes hospitaliers régionaux sont structurés par la coexistence ou la confrontation de logiques techniques et politico-administratives (départementale et régionale) dans la construction des territoires ; ils le sont également par des logiques corporatistes des fédérations et individuelles des établissements qui se manifestent dans l'activité des conférences et freinent le développement des coopérations.

Comment ces différents acteurs et ces différentes logiques s'expriment-ils au niveau plus général de l'élaboration des SROS ?

\section{L'élaboration des SROS de troisième génération}

Les SROS 3 mettent en jeu une pluralité d'acteurs. Nous avons constaté lors de la réalisation des 6 monographies régionales que ce ne sont pas les mêmes acteurs qui font ou défont les SROS. Deux principaux modes d'élaboration des SROS se dégagent:

\footnotetext{
${ }^{15}$ Entretien avec un cadre de l'ARH Nord-pas-de-Calais
} 
d'une part, un mode de gestion concertée, négociée avec les différents acteurs régionaux, en particulier avec les élus et, d'autre part, un mode de gestion à dominante technique et gestionnaire, finalement contraint par les contre-pouvoirs régionaux à prendre en compte, au moins formellement, le point de vue des acteurs locaux. Les quelques cas de confrontations de l'ARH aux contre-pouvoirs régionaux (I) contrastent avec les cas plus fréquents de gestion consensuelle et d'action de l'ARH en fonction de fenêtre d'opportunité (II). Que ce soit dans le calme ou dans la crise, les ARH sont conduites à articuler les différentes logiques techniques, financières et politiques à l'œuvre dans l'élaboration des SROS. La gestion consensuelle ou dans le calme des processus SROS fait écho aux modes de pilotage, par interaction et dans une certaine mesure par leadership, repérés dans des travaux antérieurs (Valette, 2001). La gestion conflictuelle ou dans la crise de l'élaboration des SROS III renvoie quant à elle davantage à un mode de pilotage technocratique et administré.

\subsection{La confrontation de I'ARH aux contre-pouvoirs régionaux : l'exemple du Nord-Pas-de-Calais et de Poitou-Charentes}

Dans l'ensemble des régions, l'ARH doit composer avec différents acteurs régionaux, tels que les élus locaux, les fédérations d'établissements ou encore les services de l'Assurance maladie ou de l'État avec lesquels des divergences peuvent aussi apparaître. Toutefois, dans le cadre du bilan national des SROS précité, le blocage de l'élaboration du SROS 3 est seulement apparu dans 3 régions. Dans ces régions, des coalitions d'acteurs régionaux se sont constituées pour s'opposer au mode d'élaboration du SROS par l'ARH. Ces oppositions ont porté sur le redécoupage des territoires de santé en Picardie, sur la conception de l'aménagement du territoire sanitaire et l'avenir des plateaux techniques en Poitou-charentes, et enfin sur le mode de communication de l'ARH en Nord-Pas-de-Calais.

Dans la mesure où la Picardie ne faisait pas partie des six régions sélectionnées dans notre enquête de terrain qualitative, la situation de la Picardie sera simplement esquissée. Les cas du Nord-Pas-de-Calais où la FHF s'est posée en tant que chef de file de l'opposition au SROS, puis du Poitou-Charentes où le Conseil régional est apparu comme le leader du mouvement de contestation régionale seront plus largement développés.

En Picardie, le blocage du processus d'élaboration du SROS est intervenu au stade du redécoupage des territoires sanitaires. Fondé sur la transformation des 8 secteurs sanitaires en 4 territoires de santé dont deux à cheval sur deux départements, le pré-projet de territoires de santé présenté par l'ARH a suscité une levée de boucliers des élus, à l'origine du départ de la directrice de l'ARH et du directeur médical ${ }^{16}$. La reprise du projet par un nouveau directeur et l'intervention du ministre de la santé ont finalement permis de mettre un terme à « la cabale des maires » et de faire valider la nouvelle carte des territoires de santé par les conférences sanitaires de territoire ${ }^{17}$. En Poitou-Charentes et en Nord-Pas-de-Calais, les compléments d'enquête ont permis de mettre à jour les jeux d'acteurs à l'origine de la remise en cause des SROS 3.

\footnotetext{
${ }^{16}$ Entretien avec un cadre de I'ARH Picardie.

${ }^{17}$ Entretien avec un cadre de l'ARH Picardie.
} 


\subsubsection{La FHF, chef de file de l'opposition au SROS dans le Nord-Pas-de-Calais}

La région est constituée de deux départements ; elle comporte de grandes aires urbaines et connaît une problématique de restructuration des grands établissements. Elle pâtit de mauvais indicateurs de santé (cf. annexe), ce qui constitue un argument souvent utilisé pour fonder ou justifier les programmes d'actions et les stratégies d'opposition et de revendication des différents acteurs régionaux.

\section{La contestation}

Malgré la tradition d'intervention du Conseil régional dans le domaine hospitalier et surtout en matière de santé publique, c'est la Fédération Hospitalière de France (FHF) qui semble avoir été le chef de file de l'opposition au SROS 3. Détaillée dans un document intitulé, « contribution de la FHF aux réflexions sur la version 2 du SROS $3 »^{18}$, cette opposition de la FHF porte à la fois sur la méthode de l'ARH et sur le contenu du SROS.

$\mathrm{Au}$ fond, la principale critique porte sur l'absence d'analyse des besoins de santé (simple référence à la consommation de soins) et l'absence de prise en compte des retards régionaux en matière de santé. Le 8 février 2005, un communiqué de presse conjoint de la FHF, aux élus socialistes et verts membres des CST, dénonce un schéma construit sur la consommation des soins et revendique la prise en compte des besoins de santé ${ }^{19}$. Les autres acteurs régionaux rencontrés partagent également cette analyse. Par exemple,

«Le rejet du SROS, c'est d'abord la temporalité. On a eu trois mois pour élaborer

un avis... Ensuite, la méthode qui n'était pas basée sur les besoins de santé... Et

enfin l'absence de volontarisme politique, le SROS ne permettant pas de rattraper

les retards du Nord-pas-de-Calais » (responsables administratifs Conseil régional).

En définitive, cette opposition semble issue du décalage entre, d'une part, un SROS 2 accompagné de moyens financiers, dans le cadre de la politique de péréquation nationale des enveloppes régionales, déployée par un directeur d'ARH proche des élus et, d'autre part, un SROS 3 avec peu de moyens financiers (suite au passage à la T2A), porté par un directeur d'ARH, peu à l'aise avec les élus et les principaux acteurs régionaux.

Le SROS III est ainsi élaboré suivant une approche technique réglementaire. Une myriade de COTER (comités techniques régionaux) voit le jour. Les nouvelles conférences (CST) sont créées et mobilisées dans le strict respect de la réglementation; à partir de leur installation au courant du mois de novembre 2005, elles ont 45 jours pour se prononcer sur le SROS III. Finalement, en février 2006, sous l'impulsion de la FHF, les 4 conférences rejettent le SROS III, en émettant chacune un avis négatif.

"À la réception du SROS, la FHF a pris l'initiative de réunir les principaux élus.

Elle leur a démontré que le SROS tel qu'il était présenté inversait le calendrier...

On a donc incité les élus des 4 conférences à s'opposer au SROS 3 (...) La FHF a été un des moteurs du refus du SROS» (représentant FHF).

Les conférences sanitaires de territoire (CST) dénoncent notamment le manque d'investissements dévolus aux hôpitaux publics et regrettent « la consultation à marche forcée » et

\footnotetext{
${ }^{18}$ Contribution de la FHF aux réflexions sur la version 2 du SROS 3.

19 EHESP, MIP 2008, groupe 27, « le rôle des élus dans l'organisation de l'offre de soins, l'exemples des conseillers régionaux et des présidents de conférence sanitaire de territoire de la région Nord-Pas-deCalais » p. 10.
} 
la tentative de «passage en force de l'administration $»^{20}$. Dans la foulée, le Conseil régional organise une conférence de presse pour dénoncer le déficit démocratique caractérisant le processus d'élaboration du $\mathrm{SROS}^{21}$.

\section{La résolution du problème}

Face à ce mouvement de contestation, une série de mesures très concrètes est mise en place au premier trimestre de l'année 2006 par l'ARH et finalement par le ministère. Une commission mixte rassemblant les représentants des différentes fédérations d'établissements et des conférences sanitaires de territoire est créée. Trois groupes de travail sur les objectifs quantifiés de l'offre de soins (OQOS), les PMT et les contrats pluriannuels et de moyens (CPOM) sont également constitués. L'ARH réalise un document de réponse aux doléances exprimées par les différents acteurs régionaux. Reprenant chacune des thématiques du SROS, elle répond à plus de 900 questions $^{22}$. Finalement, le directeur de l'Agence est remplacé une semaine avant la signature du SROS III prévue le 31 mars 2006.

"Il y a eu une pression importante de la FHF, du Conseil régional et de quelques

élus. Le remplacement du DARH a été demandé » (représentant FHP).

Dans la région Nord-Pas-de-Calais, le rôle de la FHF apparaît central dans le mouvement de contestation et la mobilisation des conférences contre le SROS puis en faveur de la nouvelle version du SROS.

"Ce refus a obligé l'ARH à revoir l'ensemble du SROS (...) D'où la mis en place d'une commission mixte à l'initiative du DARH et une vingtaine de réunions de janvier à mars 2006. Tous les items ont été revus pour arriver à un consensus. Ainsi, on a conseillé aux 4 conférences de voter la nouvelle mouture »(représentant FHF).

Animée par la FHF, l'opposition au SROS est soutenue par le Conseil régional, rélayée par les conférences et également alimentée par le CHU, tardivement intégré au comité de pilotage du SROS. Le cas présent traduit une confrontation frontale entre une démarche de projet « technique » paralysée par le souci de respect de la réglementation, et les attentes des acteurs de la sphère politique. À défaut de constituer ou d'être perçu comme un échelon de discussion et de négociation, l'ARH et son directeur sont contestés par les principaux acteurs régionaux et remis en cause par le ministère qui décide du remplacement du directeur de l'ARH une semaine avant la signature du SROS. Sous la pression des acteurs locaux, rejetant l'approche technique et réglementaire du SROS, les ajustements sont réalisés par le haut, par l'intermédiaire du ministère, afin de relancer la dynamique de négociation régionale.

En Poitou-Charentes, on retrouve également une forte tension entre le politique et le technique ; mais ce n'est pas exactement la même configuration d'acteurs qui entraîne le blocage du processus d'élaboration du SROS, ni le même mode de résolution de problème qui se dessine.

\footnotetext{
${ }^{20}$ EHESP, MIP 2008, groupe 27, « le rôle des élus dans l'organisation de l'offre de soins, l'exemples des conseillers régionaux et des présidents de conférence sanitaire de territoire de la région Nord-Pas-deCalais », p. 10.

${ }^{21}$ Entretien avec le vice-président du Conseil régional.

${ }^{22}$ Cahier de modifications - Questions posées par les conférences sanitaires et réponses validées par le Comité de pilotage les 10 et 17 mars 2006, Corrections SROS III - version 2, 144 p.
} 


\subsubsection{Le Conseil régional, leader du mouvement de contestation en Poitou-Charentes}

La région Poitou-Charentes, contrairement à la précédente, ne possède pas de mauvais indicateurs de santé, mais elle se caractérise par un indice de vieillissement bien supérieur à la moyenne nationale (cf. annexe). Ainsi, elle a bénéficié au cours du SROS 2 de la péréquation nationale, jusqu'au passage à la tarification à l'activité qui a conduit à supprimer cet avantage financier. Autre caractéristique majeure : il n'y a pas d'organisation pyramidale hospitalière entre les 5 départements de cette grande région au caractère rural prononcé. Enfin, lors de l'élaboration du SROS III, l'ARH semble avoir privilégié un mode de gestion à la fois technique et descendant. Dans ce contexte propice à la mobilisation des acteurs régionaux (indicateurs de santé défavorables liés au vieillissement, fin d'un mode de financement favorable, mode de gestion à dominante technocratique et descendant), l'opposition va s'organiser principalement autour du Conseil régional.

\section{La contestation}

Soutenu par le Comité économique et sociale de région (CESR), par certaines conférences de territoire et partiellement par la FHF (en matière de coopération interhospitalière), le Conseil régional va obtenir la révision de la version initiale du SROS III élaboré sous l'égide de techniciens de l'ARH et de l'Assurance maladie, avec le soutien de la FHF (en matière de restructurations). Deux thèses s'opposent, d'une part, celle des partisans de la concentration et de la spécialisation des activités de soins, soutenue par l'Assurance maladie (CRAM, CPAM, URCAM, DRSM), certains techniciens de l'ARH et la FHF et, d'autre part, celle des défenseurs de l'aménagement du territoire et du maintien d'une offre de soins de proximité portée par le Conseil régional, le CESR, certaines conférences sanitaires de territoire, certains élus locaux et également par la Mutualité Sociale Agricole. L'année qui précède la révision du SROS va conduire l'ARH et le Conseil régional à nuancer plus ou moins leur position.

La problématique de la chirurgie et de l'avenir des plateaux techniques est effectivement au cœur des tensions territoriales et politiques. Dès 2001, elle est mise en évidence dans une étude réalisée par le Centre National d'Equipements Hospitaliers et présentée aux différents acteurs régionaux. En 2005, la restructuration des plateaux techniques est affichée comme une priorité dans le SROS en cours d'élaboration. Face à cette approche qu'il juge trop technocratique et restructurante, le Conseil régional oppose une approche politique en faveur du maintien d'une chirurgie de proximité et de l'égalité d'accès aux soins.

"La première version du SROS faite sous l'impulsion du numéro 2 de l'agence reposait sur une vision très technique, sans diagnostic global (...) Il y a eu 3 délibérations du Conseil régional pour s'opposer au SROS, des réunions communes entre le CESR et la FHF pour définir une position commune, même si les approches n'étaient pas complètement communes »(cadre Conseil régional).

Ainsi, le 19 décembre 2005, une première délibération du Conseil régional dénonce l'approche exclusivement technique prévalant selon lui dans le SROS et demande à l'ARH « un délai de six mois pour l'élaboration et la mise en œuvre du SROS III, afin de peser sur les décisions qui seront prises et de mettre en place les conditions d'un débat public ${ }^{23} »$.

${ }^{23}$ Délibération relative à l'avis du Conseil régional sur le SROS III, le 19 décembre 2005. 
Le 27 mars 2006, le Conseil régional délibère à nouveau pour formuler un avis négatif sur le projet de $\mathrm{SROS}^{24}$. Le 27 février 2006, en commission permanente, les élus régionaux s'étaient prononcés à l'unanimité contre le projet de SROS III et avaient proposé l'élaboration d'un nouveau SROS avant la fin du premier trimestre $2007^{25}$.

Plus précisément, le Conseil régional revendique « la prise en compte des spécificités régionales :

- la sous-dotation hospitalière de la région, et son caractère rural ;

- le renforcement des coopérations public/public;

- l'enrichissement des missions des hôpitaux locaux et de proximité;

- la coordination de ces hôpitaux avec les professionnels de santé libéraux et une meilleure organisation des urgences ${ }^{26} »$.

Dans ce travail de contestation, le Conseil régional est soutenu par le Conseil économique et social régional qui délibère dans le même sens. Le Conseil régional s'efforce aussi de mobiliser les conférences sanitaires de territoire par l'intermédiaire de ses représentants dans les conférences. L'opposition des conférences n'est pas complète ; trois conférences sur cinq émettent un avis négatif sur le SROS, une conférence s'abstient et la dernière formule un avis positif. Le soutien de la FHF est également partiel. La FHF partage les revendications du Conseil régional en matière de coopérations interhospitalières, mais elle soutient l'ARH dans sa stratégie de restructurations des plateaux techniques.

\section{La résolution du problème}

Organisée par le conseil régional, relayée par le CESR, certaines conférences sanitaires de territoire, et partiellement soutenue par la FHF, cette opposition conduit l'ARH à prévoir la révision du SROS III dès sa parution le 30 mars 2006. Autrement dit, le SROS III arrêté en mars 2006 contient déjà l'annonce et le calendrier de sa révision (avant le $1^{\text {er }}$ avril 2007).

Entre décembre 2005 et juillet 2006, de multiples réunions sont organisées rassemblant des représentants du Conseil régional, du Conseil économique et social régional, de la FHF et des praticiens hospitaliers. Pour les élus, l'une de ces réunions animées par l'ex-président de la commission médicale d'établissement du CHU de Poitiers en décembre 2005 provoque « un choc culturel dans la mesure où ils réalisent que tout n'est pas possible partout » ${ }^{27}$. Pour alimenter sa réflexion et analyser les besoins de santé de la population dans la région, le Conseil régional fait appel au service d'un géographe consultant. Portant sur les territoires de santé, les besoins de soins hospitaliers, l'accessibilité géographique et les urgences, ses travaux concluent que « si tout n'est pas possible partout, tout n'est pas impossible partout $»^{28}$. Le Conseil régional poursuit son travail de mobilisation et propose d'organiser des réunions d'information et de mobilisation pour les hôpitaux de proximité menacés de restructuration.

De son côté, l'ARH élargit la composition du comité de pilotage du SROS en intégrant les présidents de conférences sanitaires de territoire, des représentants des usagers, des assurés sociaux et un plus grand nombre d'élus. Le SROS est révisé en deux temps. Sous l'effet de l'action conjointe des trois fédérations en mars 2006, il est décidé de revoir les

\footnotetext{
${ }^{24}$ Délibération portant avis sur le SROS 2006-2010, le 27 mars 2006.

${ }^{25}$ Commission permanente du Conseil régional Poitou-Charentes, le 27 février 2006.

${ }^{26}$ Site du Conseil régional Poitou-Charentes, rubrique Santé le 1er octobre 2007.

27 Entretien avec une directrice du Conseil régional.

${ }^{28}$ Entretien avec une directrice du Conseil régional.
} 
objectifs quantifiés de l'offre de soins (OQOS). Cette révision se fera avec les DIM (directeurs de l'information médicale) des établissements afin d'objectiver les bases du PMSI. Parallèlement, sous la pression du Conseil régional, le SROS est révisé de façon transversale au travers de réflexions sur les hôpitaux de proximité, les hôpitaux locaux, l'accessibilité aux soins et les transports sanitaires.

Cette confrontation entre l'ARH et le Conseil régional conduit donc l'ARH à mettre en place un dispositif de concertation plus ouvert et à réviser le SROS en janvier $2007^{29}$. Au fond, cette révision ne semble pas modifier le SROS en profondeur ; elle ne modifie pas l'organisation de l'offre de soins, ni le découpage des territoires de santé. Elle le complète sur les thématiques mises en avant par le Conseil régional. Inversement, cette confrontation amène le Conseil régional à nuancer son approche de l'aménagement du territoire sanitaire. Au départ sans concession, sa stratégie de défense de l'offre de soins de proximité apparaît finalement plus nuancée. Le 29 janvier 2007, le Conseil régional se prononce en faveur de la nouvelle version du SROS, sous réserve notamment de la mise en place d'une démarche d'évaluation et de suivi du SROS 3 à partir d'indicateurs des besoins de santé et non simplement de l'offre de soins. Au-delà du SROS, le Conseil régional s'engage à soutenir financièrement le développement de la télémédecine et l'installation des médecins dans les zones rurales (bourses pour les étudiants, aides à l'installation, maison de santé pluridisciplinaire).

Les ajustements réalisés par l'ARH en réponse à ce mouvement de contestation externe suscitent des réactions internes. Dans la mesure où le SROS n'affiche pas explicitement ses ambitions de restructurations, ce sont les représentants de l'Assurance maladie qui s'opposent au SROS. Au moment du vote sur le SROS à la COMEX (commission exécutive de l'ARH), les représentants de l'assurance maladie s'abstiennent; cette abstention traduit en fait une opposition de principe sur le mode de conduite des restructurations.

"Le SROS ne règle pas le problème de la chirurgie tel que nous le souhaitons. Pour cette raison, on n'a pas voté pour les deux SROS, en Poitou-Charentes et en Limousin (...) Il y a deux écoles : soit on indique clairement dans les SROS ce qui va fermer, soit on laisse les choses se faire au fur et à mesure et on laisse les plateaux mourir de leur bonne mort » (responsable service assurance maladie) ${ }^{30}$.

Dans son travail de déclinaison des orientations nationales, l'ARH est inévitablement conduite à s'appuyer sur les recommandations des services de l'Assurance maladie et de l'État (DRASS, DDASS), mais aussi à intégrer le point de vue des représentants des fédérations d'établissements et des élus. À l'exemple des régions Nord-Pas-de-Calais et Poitou-charentes, ce travail de régulation s'est fait de façon contraignante. Toutefois, à la différence de la région Nord-Pas-de-Calais, en Poitou-Charentes, le niveau régional est parvenu à conserver une certaine autonomie dans la gestion et la régulation du mouvement de contestation. Le déblocage du processus de planification a essentiellement relevé d'une régulation horizontale, au niveau régional par l'intermédiaire de l'ARH. La régulation

\footnotetext{
${ }^{29}$ Trois directeurs d'agence se sont succédés dans cette région au cours du SROS 3. Contrairement au cas du Nord-pas-de-Calais, les départs des deux premiers directeurs n’apparaissent pas directement liés au mouvement de contestation du SROS. Ils semblent essentiellement liés à des motifs personnels.

${ }^{30}$ De la même façon, les représentants de l'Assurance de maladie avait déjà manifesté leur désaccord sur la stratégie de restructuration, contenue dans première version du SROS, par une abstention au moment du vote en mars 2006.
} 
ne s'est pas faite par le haut, du moins pas explicitement, l'ARH ayant pris les mesures permettant de pacifier le processus d'élaboration du SROS. Extrêmement révélateurs et instructifs, ces modes de gestion conflictuels sont cependant assez exceptionnels ; dans la plupart des cas, l'élaboration des SROS intervient de façon plus consensuelle.

\subsection{La gestion consensuelle en fonction des fenêtres d'opportunité : l'exemple de la Haute-Normandie}

Cette petite région, composée de deux départements, est dotée d'indicateurs de santé peu favorables. Elle se caractérise par un problème majeur de démographie médicale et paramédicale, le département de l'Eure étant souvent qualifié de « désert médical ». En matière hospitalière, un important travail de restructuration des plateaux techniques a été réalisé au cours des SROS 1 et 2. «La Haute Normandie comptait 36 établissements hospitaliers pratiquant la chirurgie (dont 20 cliniques) en 1994, elle n'en compte plus maintenant que 20 (dont 15 cliniques) ${ }^{31}$. Selon un cadre de l'ARH, "le processus de recomposition de l'offre de soins s'est engagé rapidement au sein des structures privées, motivées par l'intérêt économique en premier lieu. Dans le secteur public le mouvement fut plus lent, l'argument « sécurité des soins » ne suffisant pas à faire accepter les restructurations d'établissements par les élus et la population $»^{32}$. Dans l'Eure, l'enjeu est de poursuivre la transformation des centres hospitaliers des petites villes en hôpitaux locaux ; en Seine-Maritime, l'enjeu porte davantage sur la restructuration des grands établissements, tels que le Groupe Hospitalier du Havre, le CHU de Rouen et le $\mathrm{CH}$ de Dieppe. Enfin, à la différence des Conseils régionaux du Nord-Pas-de-Calais et du Poitou-Charentes, le Conseil régional se situe en simple observateur de l'évolution de l'organisation de l'offre de soins et refuse de s'y impliquer. Dans ce contexte, l'ARH s'est efforcée d'articuler les différents points de vue techniques et politiques sur un mode consensuel et en fonction de fenêtre d'opportunité.

Un important travail d'information des élus a lieu au moment de l'élaboration du SROS 3. Les travaux sont directement présentés auprès des Conseils généraux et du Conseil régional. Un comité régional consultatif (CRC) associant notamment des représentants des collectivités territoriales et des socioprofessionnels (issu du Conseil économique et social régional) est également réuni à sept reprises entre 2004 et 2006.

Au-delà de ce travail d'information et de communication, l'ARH procède à un travail d'arbitrage entre les dimensions techniques et politiques. Par exemple, à l'occasion du passage des secteurs sanitaires aux territoires de santé, un toilettage des découpages est réalisé. Les 4 secteurs sanitaires sont globalement maintenus et transformés en 4 territoires de santé, alors que l'un de ces territoires connaît d'importants taux de fuite et que d'autres acteurs revendiquent la création d'un cinquième territoire.

"Les territoires sont basés sur le découpage du SROS précédent. L'ARH l'a adapté

à la marge (...) Malgré la faible population du territoire de Dieppe, ce territoire a été maintenu. Les élus se sont mobilisés pour le maintenir; il y a eu le temps du

${ }^{31}$ EHESP, MIP 2009, groupe 7, « Des agences régionales de l'hospitalisation aux agences régionales de santé. Bilan d'action et perspectives d'évolution à partir de l'exemple de la Haute-Normandie », p. 13.

${ }^{32}$ EHESP, MIP 2009, groupe 7, « Des agences régionales de l'hospitalisation aux agences régionales de santé. Bilan d'action et perspectives d'évolution à partir de l'exemple de la Haute-Normandie », p. 14. 
débat avec l'ARH. D'un autre côté, elle a tenu bon pour que Elbeuf, qui réclamait un territoire, ne soit pas un cinquième territoire » (représentant d'une conférence sanitaire de territoire).

Ces arbitrages se sont ensuite matérialisés dans les objectifs quantifiés de l'offre de soins (OQOS) afin de tenter de préserver les territoires de santé fragiles.

«Sur Dreux-Vernon et Dieppe, les OQOS retenus sont supérieurs aux activités constatées. C'est une manière d'afficher la place qu'on donne à ces hôpitaux dans le système.... Au contraire sur le territoire de Rouen, on a plafonné les OQOS. C'est l'aspect volontariste...» (médecin services Assurance maladie).

En matière de restructuration, certains exemples révèlent également les négociations qui ont lieu entre l'ARH et les acteurs locaux ; ils traduisent le souci d'articulation des logiques techniques et politiques. «L'exemple de la fusion des maternités des centres hospitaliers d'E. et de L. illustre ces freins politiques. Ces deux établissements possédaient une maternité et des activités de chirurgie. Le site de L. a négocié la fermeture de son service chirurgie à condition que sa maternité soit maintenue. L'ARH du fait des pressions politiques a accepté tout en sachant cette maternité condamnée. Après six ans, le constat de la non permanence des soins est devenu évident, ce qui a permis à l'ARH d'atteindre l'objectif initial de fermeture $»^{33}$. À l'image du préfet qui ne semble pas s'adresser aux élus de la même façon selon qu'il s'agit d'un maire de grande ville, d'un président de Conseil général, d'un président du Conseil régional ou d'un maire de petite communes (Michel, 1998, 1999), le directeur de l'ARH semble aussi conduit à prendre en compte le statut et l'influence politique des élus. Par exemple, la fermeture de la maternité d'une commune de taille moyenne a été réalisée par étape. Face à la résistance du maire de cette commune, également président du conseil général, cette fermeture a été obtenue en contrepartie de la mise en place d'une maison périnatale dans le cadre d'une expérimentation ; puis au terme de cette expérimentation jugée non concluante, la maison périnatale a été fermée en contrepartie de la création d'un SMUR.

L'échelon régional semble effectivement constituer un réel niveau de négociation. L'équipe dirigeante de l'ARH Haute Normandie insiste sur la nécessité de ce travail d'ajustement des objectifs de modernisation du système hospitalier avec les préoccupations et revendications locales. En ce sens, l'ARH intègre en particulier la gestion du temps politique et le statut des élus dans la planification et les restructurations sanitaires.

«Il y a des fenêtres de tirs pour les restructurations. On sait qu'en période d'élection, le ministère nous demande de ne pas intervenir; c'est inscrit dans les textes et on essaie vraiment de le respecter. Par exemple, le directeur de l'ARH ne participe à aucune inauguration pendant cette période; il évite les contacts avec les élus ; mais ça dépend aussi de l'importance des élus... Les restructurations se font au nom de la qualité et de la sécurité des soins en tenant compte des contextes politiques. Notre rôle, c'est de rendre acceptable les restructurations... Nous ne sommes pas favorables à une régulation purement économique » (cadre ARH).

L'ARH intègre aussi la capacité des acteurs locaux à intervenir directement auprès du ministère pour faire valoir leurs projets ou défendre leurs intérêts. Si l'intervention

33 EHESP, MIP 2009, groupe 7, « Des agences régionales de l'hospitalisation aux agences régionales de santé. Bilan d'action et perspectives d'évolution à partir de l'exemple de la Haute-Normandie », p. 23 et 24. 
du ministère peut effectivement fragiliser ou remettre en cause l'ARH et son directeur, la possibilité ou la «menace » de cette régulation par le haut a pour effet de renforcer l'ARH en tant qu'échelon de négociation.

"Il y a eu beaucoup de concertation. Au fur et à mesure des travaux, cela a permis la réduction des sujets de fractures. La présentation des avancées des groupes s'est faite devant les différentes instances (...) Je n'ai pas suivi le rythme de restructuration soutenu que proposaient les groupes de travail. Si les restructurations avaient continué, je n'aurai pas tenu jusqu'à la fin pour les maintenir. Il y aurait eu des réactions du terrain au ministère. La gestion du temps est un facteur essentiel » (directeur ARH).

En prenant en compte la dimension politique, l'ARH définit des lignes de conduite qui ne correspondent pas complètement ou systématiquement aux critères techniques des différents services de l'Assurance maladie.

"Il n'y a pas de fermeture prévue sur le public pour l'instant. À Gisors, Bernay, Lillebonne-Fécamp, des questions se posent.... On a une position plus volontariste visant à les restructurer avec des adaptations... Il faut éviter les doublons, partager les activités et assurer une organisation concertée public-privé pour la permanence des soins (...) Il y a des différences d'appréciation avec l'ARH qui doit gérer d'autres contraintes... On est technique. On a géré les trois quarts des groupes projets, on a fait des propositions techniques » (médecin services Assurance maladie).

Malgré ce travail d'intégration du temps et des logiques politiques, l'ARH n'est pas à l'abri des interventions directes auprès du ministère. Suivant un mode de fonctionnement stratégique et/ou strictement commercial, des établissements de santé peuvent directement faire valoir leur projet au niveau central.

"Par exemple, un ancien ministre a soutenu la constitution d'un hôpital privé avec la Générale de Santé. Le directeur de l'ARH a été court-circuité ; et il est directement allé au ministère» (cadre ARH).

En combinant les logiques technico-financières et politiques, en réalisant un arbitrage entre les recommandations de ses services techniques (Assurance maladie et services de l'État) et les revendications des autres acteurs (élus, fédérations d'établissement...), l'ARH agit afin de rendre les restructurations localement acceptables. L'ARH traduit les politiques nationales d'organisation de l'offre de soins en fonction des contextes socio-économiques et politiques régionaux. En Haute-Normandie, une approche mesurée des restructurations se dessine en fonction de la capacité d'action et de négociation des élus, de la structure de l'offre de soins, et des exigences d'efficience, de qualité et de sécurité.

"Le risque dans une petite région, c'est de tout concentrer sur Rouen, en dehors du Havre. En termes d'aménagement du territoire, c'est très clairement impensable. Le désir de restructuration de l'Assurance maladie repose sur le souci de la mấtrise des dépenses. Ils considèrent que tout doit aller dans le sens de la moindre dépense. Les élus locaux ne sont pas sur cette position. Il y a eu une grosse montée aux créneaux sur des hôpitaux de taille moyenne, comme à Gisors; les élus montent au créneau et l'ARH est prudente. D'un point de vue technique, on peut fermer Gisors mais les préoccupations de proximité et d'économie générale de l'offre sont à prendre en compte : il y a des points de frictions. L'ARH prend en considération le fait que l'offre est inférieure à la moyenne nationale; ce sont déjà des territoires 
très démunis en offre de proximité ; et par ailleurs il y a une surmortalité et une surmorbidité...» (conseiller régional).

L'exemple de la Haute Normandie illustre les modes de gestion concertée, négociée et stratégique qui semblent se dessiner dans de nombreuses régions françaises. La concertation permet l'expression d'un grand nombre d'acteurs régionaux. Au travers des groupes de travail techniques largement pilotés par des représentants de l'Assurance maladie, le point de vue des professionnels et des experts s'est exprimé. Dans le cadre des travaux de présentation du SROS aux Conseils généraux, au Conseil régional et au comité régional consultatif (CRC), les élus semblent aussi avoir été intégrés. Enfin la stratégie de restructuration en fonction de fenêtre d'opportunité traduit concrètement le travail d'articulation des logiques techniques, financières et politiques réalisé par l'ARH. À l'inverse, les exemples du Nordpas-de-Calais et du Poitou-Charentes montrent qu'à défaut d'être réalisés dans le calme, l'articulation des différentes logiques de concentration et de proximité se fait au contraire dans la crise, l'ARH étant contrainte par les contre-pouvoirs régionaux à cette régulation.

\section{Conclusion}

La permanence du politique, treize ans après leur création, les ARH ont manifestement été rattrapées par les logiques politiques auxquelles elles étaient censées échapper. Ont-elles cependant été absorbées et neutralisées par les systèmes politico-administratifs locaux ? Le découpage des territoires de santé de recours est encore souvent structuré par les territoires départementaux et régionaux. Les modes d'élaboration des SROS montrent aussi clairement l'importance du rôle joué par certains conseils régionaux et globalement par les élus locaux. Les ARH doivent effectivement composer avec les élus, mais pas seulement. De multiples acteurs porteurs de logiques différentes s'y expriment ; et il revient aux ARH de rendre acceptable les restructurations hospitalières auprès de ces différents acteurs. En ce sens, on ne peut pas parler d'absorption, ni de neutralisation des ARH par les acteurs locaux. En fait, les ARH intègrent le point de vue et les contraintes des élus locaux à la gestion régionale des affaires sanitaires.

L'ARH a un rôle d'articulation volontaire ou forcée des logiques technico-financières et politiques. Que ce soit au niveau des territoires de santé ou de l'élaboration des SROS III, l'ARH est conduite à prendre en compte de multiples stratégies. Ainsi les différentes politiques régionales de l'organisation de l'offre de soins semblent reposer sur un compromis entre les différentes logiques d'action qui se côtoient ou s'affrontent dans les régions :

- les logiques techniques et financières portées par l'Assurance maladie (DRSM, CRAM, URCAM) en faveur de la concentration et de la spécialisation des activités au nom des exigences de qualité-sécurité et d'efficience;

- la logique de santé publique défendue par les DRASS, susceptible de remettre en cause la primauté des établissements de santé dans l'organisation de l'offre de soins ;

- les logiques corporatistes plutôt favorables à la concentration et à la spécialisation des activités défendues par les différentes fédérations d'établissement;

- les logiques individualistes développées par les différents établissements de santé qui souhaitent défendre ou développer leurs parts de marché dans un système de soins financés par la T2A; 
- les logiques politiques déployées par des élus oscillant entre réflexes localistes de défense des emplois locaux et conceptions régionales de l'aménagement du territoire sanitaire.

De façon spontanée ou contrainte, l'ARH réalise un travail d'articulation de ces différentes stratégies et logiques en vue d'une restructuration plus ou moins progressive des établissements de santé et de l'offre de soins régionale.

La régulation horizontale régionale est renforcée par la « menace » de la régulation verticale ministérielle. Même si les risques de court-circuitage persistent, les ARH semblent constituer un niveau d'arbitrage relativement stable entre les différentes approches techniques, corporatistes et politiques qui s'expriment au niveau local. Le fonctionnement des ARH consacre la consolidation d'un niveau de régulation régionale initiée par les DRASS au début des années 1990. Avec les SROS 3, une relative autonomie de gestion régionale se dessine autour de l'acteur « gestionnaire public » (l'ARH), impliquant les techniciens (des services déconcentrés de l'État et de l'assurance maladie), les corporations professionnelles et les élus. L'échelon régional s'impose progressivement comme un niveau effectif de régulation horizontale entre les différents acteurs locaux. L'action collective et les négociations tendent à s'institutionnaliser au travers des différentes étapes et instances de gestion de projet générées par la mise en œuvre des SROS 3, mais aussi de la contractualisation avec les établissements.

Toutefois, la persistance des stratégies de contournement des ARH, notamment par les élus pour faire directement pression au ministère, témoigne aussi de l'existence d'un mode de régulation verticale qui rappelle la régulation croisée. Malgré des airs de famille, cette régulation de type vertical ne peut être assimilée à la régulation croisée qui constituait le principal mode d'ajustement des décisions centrales dans le système départemental antérieur à la décentralisation. Actuellement, ces ajustements «par le haut » semblent essentiellement complémentaires de la majeure partie ${ }^{34}$ des ajustements réalisés au niveau régional par les ARH.

Dans le domaine hospitalier, les arbitrages nationaux peuvent remettre en cause les instances et les décisions régionales ou imposer certains projets. Simultanément, la possibilité et la «menace » de cette régulation par le haut semblent aussi pousser les ARH à intérioriser la nécessité d'une gestion concertée de l'organisation de l'offre de soins. Elles semblent inciter les ARH à une plus large institutionnalisation de l'action collective au niveau régional. La régulation se fait de plus en plus à un niveau horizontal et territorial par l'intermédiaire des ARH ; mais de façon complémentaire, elle peut aussi être verticale par l'intermédiaire des pressions politiques exercées directement auprès du ministère. Plus précisément, dans une perspective de co-construction locale de l'action publique à partir d'orientations et de cadres nationaux, la régulation verticale complète et conforte par «sa menace » la régulation horizontale régionale, assurée de façon plutôt consensuelle et/ou en fonction de fenêtre d'opportunité par les ARH.

Ce mode de régulation à dominante régionale, sous la pression potentielle d'arbitrages nationaux, permet une diffusion progressive des logiques financières et médico-techniques

\footnotetext{
${ }^{34}$ II serait effectivement intéressant d'approfondir l'analyse de ces modes de régulation par exemple à partir de l'analyse comparée des projets aboutis ou bloqués de restructurations hospitalières dans différentes régions.
} 
soutenues par l'Assurance maladie et également le maintien d'une conception hospitalocentrée de l'organisation de l'offre de soins défendue jusqu'à présent par les fédérations, les établissements et certains élus.

Dans le cadre des ARS, la synthèse des différentes logiques techniques, politiques, corporatistes et individualistes sera au centre de l'activité des nouvelles agences. Ce travail sera cependant d'autant plus complexe qu'une articulation croissante entre les différents champs de compétence des ARS (hospitalier, santé publique, médecine de ville, médicosocial...) sera recherchée.

\section{Bibliographie}

BLANCO F., (2000), La planification française dans le domaine hospitalier, Presses Universitaires AixMarseille, Aix-en-Provence.

BORGETTO M. et LAFORE M., (2000), La république sociale. Contribution à l'étude de la question démocratique en France, PUF, Paris.

COMMAILLE J. et JOBERT B., (1999), Les métamorphoses de la régulation politique, LGDJ, Paris.

CHEVALLIER J., (2004), L'État régulateur, Revue française d'administration publique 111, 473-482.

COLDEFY M. et LUCAS-GABRIELLI V.,(2008), Les territoires de santé : des approches régionales variées de ce nouvel espace de planification. Institut de recherche et documentation en économie de la santé, Document de travail, DT n ${ }^{\circ} 10$.

CROZIER M. et THOENIG J.C., (1975), La régulation des systèmes organisés complexes. Le cas du système de décision politico-administratif local en France, Revue française de sociologie 16, 3-32.

DENIS J.L. et VALETTE A., (1997), La régulation régionale au concret : l'expérience des DRASS, Politiques et management public, 15 (4), 1-25.

DENIS J.L., FARGEON V., MINVIELLE E. et VALETTE A., (2001), Histoire et bilan d'une innovation institutionnelle: les agences régionales de l'hospitalisation. Convention INSERM/MIRE/CNRS Décision $n^{\circ} 4 \mathrm{M} 615 \mathrm{E}$.

DURAN P. et THOENIG J.C., (1996), L'État et la gestion publique territoriale, Revue française de science politique 4, 580-623.

FARGEON V., MINVIELLE E., VALETTE A. et DENIS J.L., (2002). Les agences régionales de l'hospitalisation ont cinq ans : bouleversement ou aménagement du dispositif de régulation? Politiques et management public 20 (2), 43-60.

GAMBLE A., (1994), The free economy and the strong State, Macmillan Press, London.

JOURDAIN A. et DUMOND J., (2006), L'action publique entre la planification et la contractualisation négociée : la perception des acteurs politiques locaux, Politiques et management public 24(2), 1-21.

JOURDAIN A. et BRECHAT P.H., (2008), La nouvelle planification sanitaire, Les éditions de l'EHESP, Rennes.

KERLEAU M., (2003), L'ARH: un espace de négociation pour les restructurations hospitalières ?, Revue française des affaires sociales 3, 30 .

LE GALES P. et SCOTT A., (2008), Une révolution bureaucratique britannique ?, Revue française de sociologie 49 (2), 301-330.

MAJONE G., (1997), From the positive to the regulatory Sate: causes and consequences of the change in the mode of governance, Journal of public policy 17 (2), 139-167.

MAJONE G., (1999), The regulatory State and its legitimacy problems, West European Politics 22, 1-24.

MAQUART B., (1996), La réforme de l'hospitalisation publique et privée, Droit social 9/10, 869.

MICHEL H., (1998), Government or governance? The case of the French local political system, West European Politics 21, 146-169. 
MICHEL H., (1999), Intercommunalités et gouvernements locaux, L’Harmattan, Paris.

MICHEL H. et BELLANGER M., (2002), La régionalisation sanitaire et médico-sociale entre enjeux étatiques, pouvoirs locaux et construction européenne, article présenté au congrès économétrie de la santé XIV: santé et régionalisation, Ajaccio 10-11/10/2002.

POLANYI K., (1983), La grande transformation, Gallimard, Paris.

SCHWEYER F.X.,(1998), La régulation régionale du système hospitalier: pilotage par l'État ou reterritorialisation ?, Politiques et management public 16 (3), 43-68.

THOENIG J.C., (1999), L'usage analytique du concept de régulation in Commaille J. et Jobert B. Les métamorphoses de la régulation politique, LGDJ, Paris.

VALETTE A., (2001), Fallait-il une nouvelle organisation pour changer les modes de régulation? L'expérience des agences régionales de l'hospitalisation, Revue française des affaires sociales 55(4), 69-77.

VOGEL S., (1996), Freer markets, More rules, regulatory reform in advanced industrial countries, Cornell University Press, Ithaca and London. 
Annexe 1 - Profil des régions étudiées

\begin{tabular}{|c|c|c|c|c|}
\hline Région & Nord-Pas-de-Calais & Poitou Charente & Haute-Normandie & France \\
\hline $\begin{array}{l}\text { Territoire } \\
\text { et graduation } \\
\text { des soins }\end{array}$ & recours + proximité & $\begin{array}{l}\text { recours }+ \\
\text { proximité }+ \\
\text { intermédiaire }\end{array}$ & $\begin{array}{l}\text { recours + } \\
\text { proximité }\end{array}$ & \\
\hline $\begin{array}{l}\text { Approche } \\
\text { thématique } \\
\text { (intégrée, orientée } \\
\text { ou règlementaire) }\end{array}$ & intégrée & intégrée & Orientée & \\
\hline Réaction politique & processus affecté & processus affecté & $\begin{array}{l}\text { processus } \\
\text { non affecté }\end{array}$ & \\
\hline PMT & non réalisés & non réalisés & non réalisés & \\
\hline $\begin{array}{l}\text { CPOM dans } \\
\text { les délais? }\end{array}$ & non & oui & oui & \\
\hline $\begin{array}{l}\text { Densité au } \\
1 / 01 / 2006 \\
\text { en hab / km² }\end{array}$ & 326 & 66 & 147 & 112 \\
\hline $\begin{array}{l}\text { Taux comparatif } \\
\text { de mortalité } \\
\text { en } 2006 \\
\text { Hommes } \\
\text { Femmes }\end{array}$ & $\begin{array}{l}376 \\
158\end{array}$ & $\begin{array}{l}270 \\
116\end{array}$ & $\begin{array}{l}314 \\
133\end{array}$ & $\begin{array}{l}270 \\
120\end{array}$ \\
\hline $\begin{array}{l}\text { Indice de } \\
\text { vieillissement } \\
\text { au 1.01.2006 }\end{array}$ & 51,2 & 89,7 & 58,5 & 66 \\
\hline $\begin{array}{l}\text { Lits pour } \\
1000 \text { habitants } \\
\text { (médecine hôpital) }\end{array}$ & 2,01 & 1,91 & 1,91 & 2,11 \\
\hline $\begin{array}{l}\text { Lits pour } \\
1000 \text { habitants } \\
\text { (chirurgie hôpital) }\end{array}$ & 1,58 & 1,46 & 1,42 & 1,62 \\
\hline $\begin{array}{l}\text { Soins de suite pour } \\
1000 \text { habitants } \\
\text { (équipement } \\
\text { global) }\end{array}$ & 1,41 & 1,41 & 1,31 & 1,60 \\
\hline $\begin{array}{l}\text { Médecins } \\
\text { généralistes pour } \\
100000 \text { habitants }\end{array}$ & 113 & 113 & 99 & 112 \\
\hline
\end{tabular}




\section{Annexe 2 - La structuration départementale des territoires de santé de recours}

Le découpage des territoires de santé peut être qualifié de technique dans 11 voire 12 régions (Nord-pas-de-Calais, Champagne-Ardenne, Lorraine, Alsace, Franche-Comté, Picardie, Rhône-Alpes, PACA, Bretagne, Basse-Normandie, Auvergne et enfin Haute-Normandie). Par exemple, dans la région Nord-Pas-de-Calais composée de deux départements, le périmètre des 4 territoires de santé de recours a été délimité suivant une logique technique et afin « de casser les approches départementales » ${ }^{35}$. Par ailleurs, 4 des 5 territoires de santé sont interdépartementaux en Champagne-Ardenne, 2 sur 4 en Picardie, 3 sur 5 en BasseNormandie et en particulier le territoire du sud-est, 3 sur 6 en Franche-Comté, 5 sur 8 en Bretagne, 6 sur 9 en PACA, 11 sur 14 en Rhône-Alpes. S'agissant de la Haute-Normandie, le découpage apparaît un peu atypique. Dans cette région, les frontières départementales sont effectivement débordées par les 4 territoires de santé ; cependant, pour des raisons de politiques locales et non plus départementales - qui seront développées dans la deuxième partie de cet article- l'un des territoires, celui de Dieppe, est maintenu alors qu'il connaît des taux de fuite extrêmement importants. Enfin, en Alsace, les 4 territoires de santé agencés suivant une logique de stratification de la région du nord au sud débordent le cadre départemental au niveau d'un territoire de santé. En Lorraine, les 2 territoires de santé de grande envergure sont aussi interdépartementaux, en particulier dans le territoire du nord de la Lorraine.

Inversement, dans 3 voire 4 régions (Centre, Limousin, Midi-Pyrénées, Corse), le périmètre des territoires de santé de recours est strictement départemental. Ainsi dans la région Centre, les 6 territoires de santé correspondent exactement aux 6 départements. Dans le Limousin, les 3 territoires de santé respectent aussi parfaitement les frontières des 3 départements. En Midi-Pyrénées, les frontières départementales structurent également le découpage des territoires de santé. 5 territoires de santé sont strictement départementaux (Ariège, Haute-Pyrénées, Gers, Tarn-et-Garonne, Lot). 4 autres territoires de santé relèvent d'un découpage du département entre sa partie nord et sa partie sud (Tarn, Aveyron). Les 6 autres territoires de santé s'inscrivent dans le département de la Haute-Garonne. Dans ce dernier cas, on devine aisément qu'à l'intérieur de ce cadre départemental les territoires de santé ont été délimités en fonction de critères plus techniques. Globalement, dans la région Midi-Pyrénées, le cadre départemental a été, soit décomposé en plusieurs territoires de santé, soit intégralement repris pour constituer un territoire de santé. En Corse, à l'exception d'un canton rattaché au territoire sud, les deux territoires de santé reprennent les frontières départementales.

Entre ces modes de construction techniques ou départementaux, 6 régions telles que le Languedoc-Roussillon, semblent relever d'un mode de construction mixte. En LanguedocRoussillon, parmi les 8 territoires de santé, l'un d'entre eux est complètement départemental (les Pyrénées-Orientales), alors qu'un autre (celui de Montpellier) est manifestement interdépartemental. Toutefois dans ce groupe de régions au mode de construction mixte, le prisme départemental apparaît dominant (Bourgogne, Poitou-Charentes, Pays de la Loire, Aquitaine et Ille de France). En Bourgogne, parmi les 6 territoires de santé, celui de la Côtes d'Or est strictement départemental ; le département de la Saône-et-Loire est découpé,

${ }^{35}$ Entretien avec un cadre de I'ARH Nord-pas-de-Calais 
suivant une ligne de partage nord/sud, en deux territoires. En Aquitaine, dans les Pays de la Loire et en Poitou-Charentes, l'empreinte départementale est également très présente. En Aquitaine, 2 des 8 territoires de santé sont dessinés sur le patron départemental, la Gironde avec quelques cantons en plus et le Lot-et-Garonne avec quelques cantons en moins. On retrouve cela aussi dans les Pays de la Loire, avec le territoire de santé de la Mayenne, comprenant la totalité du département de la Mayenne et le canton de Segré appartenant au Maine-et-Loire. L'un des territoires est aussi strictement départemental, celui de la Sarthe. En Poitou-Charentes, les 5 territoires de santé de recours obéissent globalement à une logique départementale (Deux-Sèvres, Vienne, Charente) ou de regroupement à l'intérieur d'un département (Charente-Maritime). Enfin en Ille de France, le cadre départemental apparaît clairement dans le regroupement de 3 territoires de santé dans les Hauts-de-Seine, le Val-d'Oise, les Yvelines et à Paris, dans le regroupement de 2 territoires de santé dans la Seine-et-Marne (à l'exception d'un canton dans le nord du département) ; quelques esquisses de découpage interdépartemental existent en effet à la marge entre des territoires de santé des départements de la Seine-St-Denis et de la Seine-et-Marne (1 canton), entre ceux du Val-de-Marne et de la Seine-St-Denis (1 canton), et surtout de l'Essonne et du Val-de-Marne. 
